\title{
Performing Patient-Specific Knee Replacement with Intra-Operative Planning and Assistive Device (CAS, Robotics)
}

\author{
M. Cievet-Bonfils, C. Batailler, T. Lording, \\ E. Servien, and S. Lustig
}

\subsection{Introduction}

Computer-assisted surgery (CAS) and robotic surgery have shown promise for joint replacement by improving surgical precision regarding bone resections and ligament balance [1]. The objective is not to substitute the surgeon, but rather to assist the surgeon with the goal to perform a more precise implantation.

Data suggest that systems involving 3D preoperative planning and custom guides are being employed more frequently and benefit the precision of the implantation. The inconveniences of these systems are the need for pre-operative computed tomography (CT) scan with specific protocol and the cost for manufacturing the custom guides.

An evolution of robotic surgery has been developed and relies on a bone morphing step during surgery, such as with the NAVIO system $\left(\right.$ Smith Nephew $\left.{ }^{\circledR}\right)[2,3]$. Preoperative CT imaging is therefore no longer necessary. These systems have the potential to improve the surgical

M. Cievet-Bonfils · C. Batailler · E. Servien

S. Lustig $(\bowtie)$

Orthopaedic Department, Lyon North University

Hospital, Lyon, France

e-mail: maxime.cievet-bonfils@chu-lyon.fr; cecile.batailler@chu-lyon.fr; elvire.servien@chu-lyon.fr

T. Lording

Melbourne Orthopaedic Group,

Windsor, VIC, Australia

accuracy for knee arthroplasty. Nevertheless, the surgical steps and the potential difficulties must be understood. This chapter presents the technical aspects of this evolution, current and future applications, and surgical tips to perform robotic or computer-assisted surgery easily.

\subsection{Computer-Assisted Surgery and Robotics}

CAS and robotic surgery permit intra-operative definition of distal femoral and proximal tibial bone anatomy through the bone morphing process, as well as determination of mechanical axes (femoral, tibial and limb) and knee range of motion.

Robotic surgery permits dynamic acquisition of ligament laxity during the planning phase, assists placement of cutting guides and finally allows the evaluation of residual ligament laxities.

Implants positioning is performed accurately with the robotic system and 3D planning during surgery. With the system BlueBelt (Smith and Nephew $\left.^{\circledR}\right)$, bone resection is done by the surgeon who manipulates the handpiece, while the computer retracts the burr when the handpiece moves outside the planned bone resection zone. This specific system needs minimal preoperative imaging. We do not perform any pre-operative 3D imaging but only a standard radiographic assessment. 


\subsection{Unicompartmental Knee Arthroplasty}

Implanting femoro-tibial or patello-femoral UKAs is technically demanding, and its success depends on the quality of the indication and its implantation. This makes these procedures ideal for use of robotic technology.

\subsubsection{Medial UKA Surgical Technique}

\subsubsection{Installation}

The patient is placed in a supine position, with a lateral support and a foot wedge to maintain the knee at $90^{\circ}$ of flexion. A tourniquet can be used according to surgeon preference.

The NAVIO PFS console consists of three elements:

- An infrared camera (as in a conventional surgical navigation system) that must be installed about $1 \mathrm{~m}$ from the surgical field, facing the operator, so as to permanently visualize the femoral and tibial sensors.

- A touch screen covered with a sterile drape. It is located within reach of the operator, most often at the level of the contralateral hip.

- A console controlling the robotic burr and irrigation during resection. The handpiece can be held in one hand and is connected to the console by a cable and the irrigation tubing.

The first step is positioning of the femoral and tibial sensors, most often in a percutaneous fashion to the tibia and with a minimal subvastus approach to the femur so as not to pass through the quadriceps. These sensors must be visible throughout the procedure and throughout the range of motion of the knee. The incision is parapatellar (medial or lateral), typically from the patellar superior pole to about $1 \mathrm{~cm}$ below the joint line, over a length of about $10 \mathrm{~cm}$. It is important that osteophytes are removed before any registration by the ligament balancing system.

\subsubsection{Point of Interest Acquisition}

This stage of the procedure used to be long and tedious with older CAS systems, but advances in computing and technology have made it possible to optimize it. To ensure that the sensors are stable throughout the procedure, a reference point is identified at the tibia and femur, allowing the surgeon to check with the probe that the sensors have not moved.

The hip centre is acquired by repeated circumduction movements of the leg with a maximum permissible error of $0.9 \mathrm{~mm}$. The internal and external malleoli are acquired at the ankle directly with the probe. The full range of motion of the knee is then recorded, thanks to a complete flexion-extension movement without constraint in varus nor valgus. The same extension-flexion movement is then performed with a stress in valgus (or varus in the case of lateral unicompartmental) in order to record the reducibility of the deformation throughout the range of motion. This dynamic acquisition is essential because it allows the system to consider ligament laxity during the planning phase.

Points of interest are then acquired on the femur with the probe: the centre of the knee (at the top of the notch), the most distal, most posterior and the most anterior points of the medial condyle (which corresponds to the contact between the most anterior point of the tibial plateau and the femoral condyle in complete extension of the knee). The femoral acquisition continues with a bone morphing phase of the surface of the condyle using the probe (Fig. 26.1).

The same sequence is then repeated for the tibia: the centre of the tibia, the most distal point of the tibial cup, the most posterior point (for which access is made difficult by the intact femoral condyle), the most medial point and the most anterior point. The anteroposterior axis of the tibia is also recorded, before finishing the tibial acquisition with a phase of surface bone morphing.

\subsubsection{Planning}

This is one of the essential steps of the robotic system because it allows real-time dynamic planning, taking into account the reducibility of the deformation. 

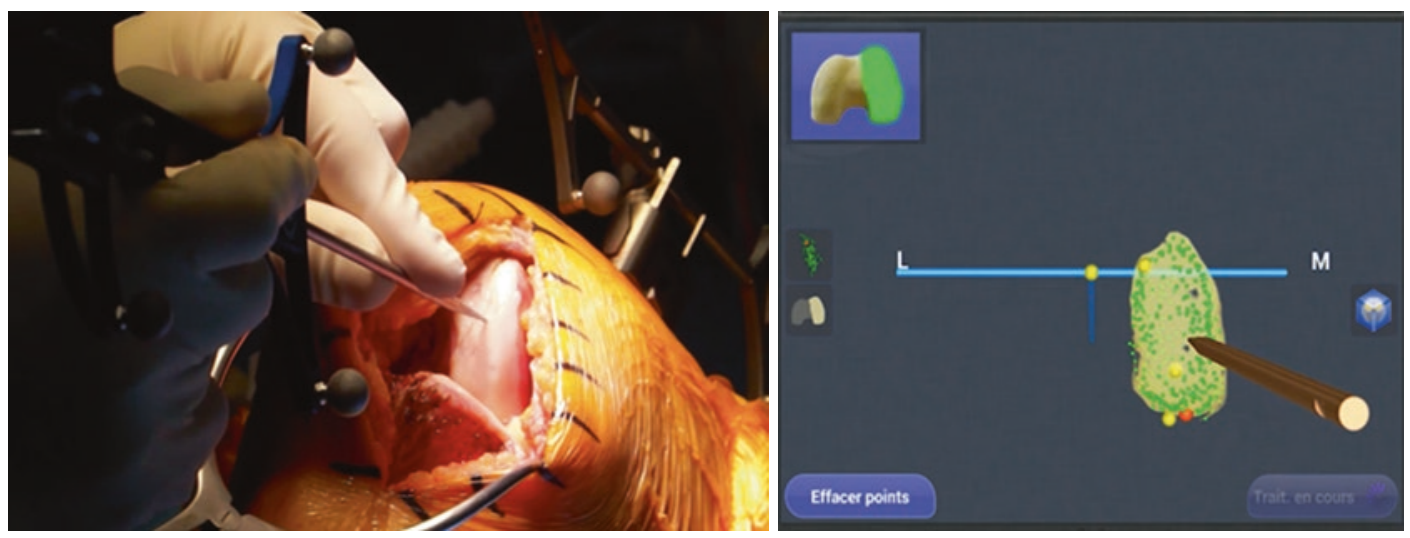

Fig. 26.1 Femoral condyle bone morphing

The first step is to choose the size of the femoral implant, which can be modified at any time during the surgery. Then we choose the position of the femoral component, within the three planes of space. A separate screen split into four parts demonstrates the exact position of the implant in relation to the shape of the femoral condyle. The touch screen allows manipulation of a 3D view of the femoral condyle with the implant planned, in order to precisely visualize the final position. The angular values of this femoral implant are visible at any time: varus/valgus, flexion and rotation.

The objective is to obtain maximal coverage of the bone surfaces, maintaining the joint line height and avoiding impingement with the massive tibial spines.

The same steps are then performed for the tibial component. We first decide the size of the implant and the thickness of the polyethylene. Then we choose the varus/valgus, rotation, tibial slope and positioning of the implant in relation to the tibial spines. The touch screen also allows rotation of 3D images to accurately visualize the positioning of the implant in the three planes of space. As always for a unicompartmental prosthesis, tibial bone resection should be minimized.

The next step is to visualize the consequences of our planning in terms of the angular correction (preoperative vs. postoperative) between $0^{\circ}$ and $120^{\circ}$ of flexion. At this stage, we can change the position of the tibial implant (varus/valgus, slope, rotation, resection depth) and femoral implant (varus/valgus, flexion, rotation, resection height) and visualize the consequences on the final angular correction. These parameters not only take into account static acquisitions but also the initial dynamic acquisitions, and therefore the reducibility of the deformation at each flexion angle (Fig. 26.2).

The final stage of planning is to visualize contact points between the components during flexion, which allows lateralization or medialization if necessary of either component to better centre this point of contact. You can freely navigate between the different planning screens. Once the desired result is obtained, the final choice is validated.

\subsubsection{Preparation of Bone Surfaces}

Once the planning is validated, we can prepare the bone surfaces. The assembly of the robotic burr with the irrigation system and the calibration phase takes a few seconds. A final control step allows you to visualize the area to be drilled. We check that it corresponds visually to the area where you want to position the implant.

We usually start with the femur which is more easily accessible. It is also possible to start with the tibia if desired. An automatic feedback system only burrs the planned area. If you leave this area, the burr is retracted, making it impossible to resect bone in an undesired area by mistake. The remaining bone depth to be removed is continuously displayed on the screen in a colour-coded fashion, which makes it possible to orient the 


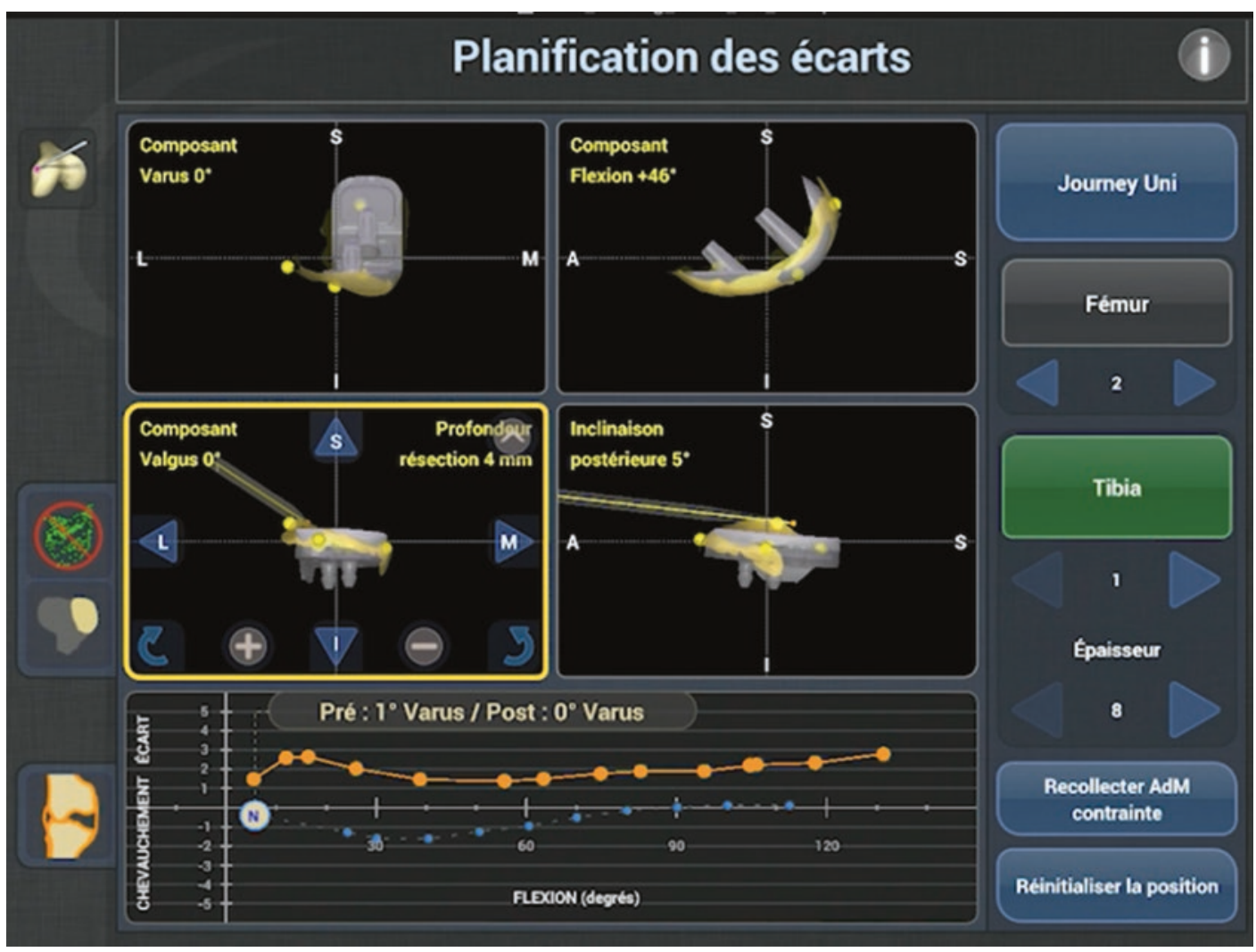

Fig. 26.2 Global knee balance planning according to the positioning of femoral and tibial implants

burr in an efficient way. The surgeon has complete freedom of movement; the robotic system only retracts the burr when it is moved outside the planned area. We gradually mobilize the knee in hyperflexion to reach the posterior femoral region. It is sometimes necessary to burr the tibia before being able to access the most posterior part of the femoral condyle.

Once the femur is prepared, we move on to the tibia with the same visual control. We begin on the most anterior part of the tibia, gradually extending to the entire planned surface. It is possible to use the most anterior part of the bone resection as a guide for a saw, and to saw the posterior part, in order to save a few minutes of operative time. A rasp is then used to file down any irregularities in the bone resections once burring is finished. The meniscus, easily accessible at this stage, is then removed. The last step consists of drilling for the anchoring lugs of the femoral implant under visual control.

\subsubsection{Trial and Final Implants}

We can then place the trial implants and display on screen the correction angle obtained as well as the ligament balance throughout the range of motion. Cementation and fixation of final implants are done according to surgeon preference. We can once again check the angular correction and balancing of the knee with the final implants in situ.

\subsubsection{Results}

In our experience with the NAVIO system, the results and particularly the positioning of the implants have been significantly improved. One of the essential parameters for the success of a knee joint prosthesis is to reproduce the joint line level [4]. Our results published on unicompartmental prostheses have shown that this parameter was very well controlled with this robotic system while giving favourable clinical results in the short and medium term since 2013 [5]. Hopefully, 
longer term results will confirm these encouraging initial radiological and clinical findings. In literature, the mean implant positioning is not significantly improved with robotic-assisted UKA. By contrast, the reduction of outliers is significant [6] and thus very relevant to reduction of failure. Ponzio and Lonner have reported that aggressive tibial resection is less frequent during robotic-assisted UKA [3].

Studies on robotic-assisted UKA report satisfying short- and medium-term survival rates [7]. Nevertheless, no comparative study has demonstrated a better survival rate for robotic-assisted UKA, compared to conventional UKA. Published rates of revision after robotic-assisted UKA vary from $3 \%$ to $10 \%$ at midterm [8-10].

\subsection{Patello-Femoral Knee Arthroplasty}

Patello-femoral prosthesis involves different difficulties. However, it is probably one of the best indications for robotic surgery, as ideal positioning requires a precise understanding of the three-dimensional anatomy of the distal femur. The bone preparation requires the use of a burr, even with conventional instrumentation. 3D planning is made easy by the acquisition phase of the NAVIO system, which produces a $3 \mathrm{D}$ model of the trochlea and records the reference axes (bi-epicondylar, Whiteside, femoral mechanical). It is thus possible to visualize the desired 3D positioning of the trochlear implant and to ensure a perfect transition of the femoral implant with the cartilage of the femoral condyles, before the bone resection. The preparation phase is facilitated by the controlled burring system with the robotic handpiece that burrs the cartilage and the subchondral bone according to the planning, in a more reproducible fashion than with standard instrumentation. Very few studies described results of robotic-assisted patella-femoral prosthesis, and these studies are not comparative. The first series reported satisfying functional scores and a good implant positioning [11].

\subsection{Total Knee Arthroplasty}

\subsubsection{Computer-Assisted Surgery}

Computer-assisted surgery was pioneered in the early 1990s, with the first total knee replacement performed in 1997. Computer navigation offers intra-operative dynamic assessment of alignment, balance and kinematics.

The computer-assisted system is composed of two elements:

- An infrared camera that must be installed about $1 \mathrm{~m}$ from the surgical field, facing the operator, so as to permanently visualize the sensors during all the surgery.

- A touch screen.

The surgery starts by placing sensors on the femur and the tibia. We then acquire the different mechanical angles and the hip centre with the use of the handpiece. A standard approach is then used to access the bone surfaces. The handpiece is used to perform bone morphing of the femoral condyles and the tibial plateau. We then place the bone cutting guide on the femur to perform the distal cut.

The bone cutting guides can be controlled in varus/valgus, flexion/extension and internal/ external rotation for both the femur and the tibia. They have sensors that allow the computer system to calculate the angles of bone cutting. When adjusting the cutting guides, the operator can see on the screen the effect it will have on the bone cut. The operator can then make the cuts following the planning on the screen. After the cuts are performed, the surgery is the same as for a traditional total knee replacement.

\subsubsection{Robotic}

Computer-assisted surgery systems help place cutting guides using anatomical and ligament balance data. The NAVIO system is an evolution using more detailed anatomical data, especially regarding the soft tissues (Fig. 26.3). In fact, acquisitions are made throughout the range of 


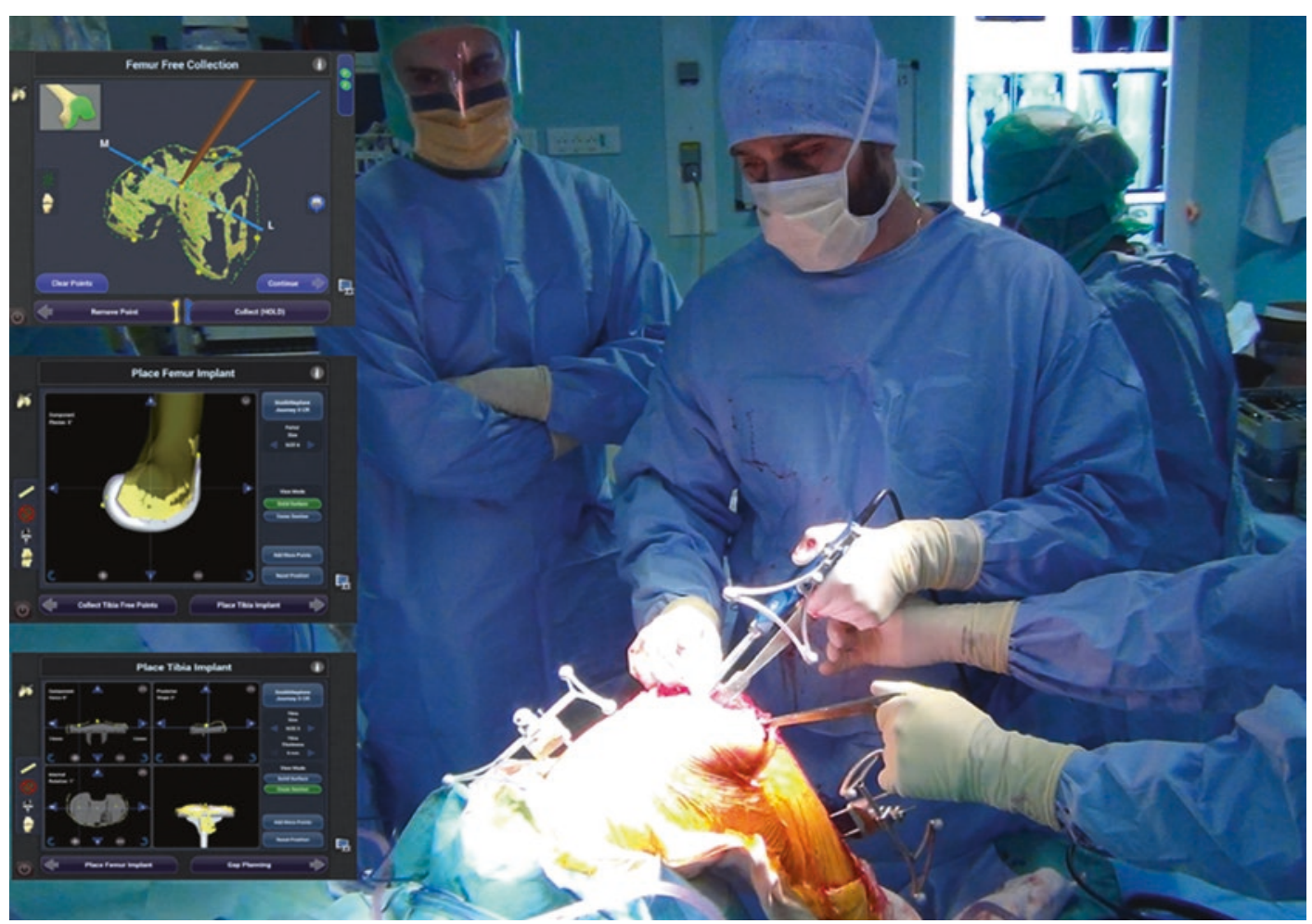

Fig. 26.3 Total knee arthroplasty with NAVIO system

motion in order to place the femoral and tibial components to optimize stability throughout the full range of motion, not only at $0^{\circ}$ and $90^{\circ}$ of flexion.

Balancing and soft tissue release can be checked and improved during the data collection phase. The alignment and the possible ligament contractions or laxities will be collected through all the range of motion.

Sizing and positioning of the components are done before any bone cutting. Virtual planning allows sizing and positioning of implants corresponding to anatomy and ligament balance. Once the planning is validated, the surgeon uses the robotic burr to make four holes in the distal femur and two to four holes in the proximal tibia. The cutting guides are placed using these holes as guides and then fixed on the bone (Fig. 26.4). Virtual cuts can be visualized on the screen before making them by placing a tool in the cutting slot. These cuts can also be checked after completion with the same tool.
The trial implants are then placed, and examination of the ligament balance through the full range of motion can be compared to the initial plan, before cementation of the final implants.

This type of programming is particularly interesting when using implants reproducing the kinematics of the knee. Indeed, they require taking into account not only the bone references but also the soft tissues in order to reproduce ideal articular kinematics. The robotic assistance allows finalizing this reflection by realizing the gesture with a suitable precision.

\subsubsection{Literature}

In a study comparing conventional versus computer-assisted surgery for TKA with 5-year follow-up, Cip et al. did not show any difference in implant survival rate, but poorer accuracy for mechanical axis and tibial slope in the conventional group. Clinical examination 


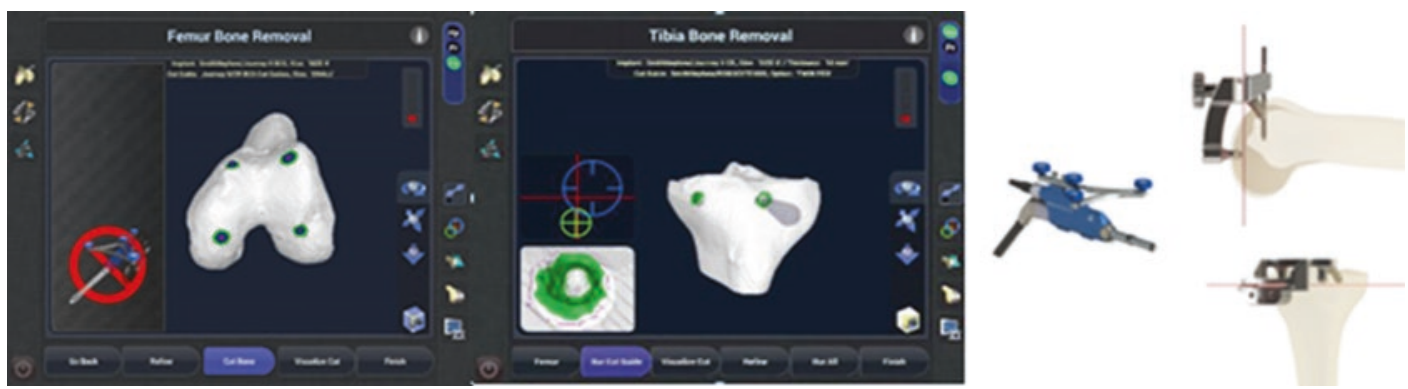

Fig. 26.4 Preparation of the femoral and tibial cutting guides' position with the robotic handpiece

showed no difference between the two groups; however, the Knee Society Score was better in the navigated group [12]. There are very few studies on robotic-assisted TKA. These studies are essentially preliminary studies without comparative group or long-term follow-up. A long-term follow-up and a randomized study are necessary to reliably assess the benefit of robotic-assisted TKA.

\subsection{What's Next?}

The success of robotic surgery systems suggests that their place in operating theatres will become more and more important. It may provide significant support for the placement of bi-cruciateretaining knee replacement prostheses, which require a thorough understanding of the different characteristics of the medial and lateral compartments, as well as precise bone preparation. The protection of the tibial spine should also be simpler with the use of a burr guided by a robotic handpiece.

Currently, a recent concept is developed for the lower limb alignment during TKA. The kinematic alignment (KA) of TKA allows to conserve a part of the knee constitutional deformity and to obtain a more physiological knee with easy ligament balancing. Several studies have reported better functional outcomes after a kinematic alignment with a better restoration of a normal gait $[13,14]$. Very few TKA ancillaries exist to perform accurately a KA. Some studies have assessed the efficiency of a robotic surgery system to obtain a satisfying KA and to compare outcomes with mechanical alignment [15-17]. In a randomized study, Yeo et al. compared the outcomes and gait analysis after robotic-assisted TKA with either a KA or a mechanical alignment [15]. After a follow-up of 8 years, they find the same results between both groups. The robotic-assisted surgery could be very interesting to perform an accurate kinematic alignment during TKA. This use is still uncommon with few studies. More studies are needed to assess the potential of robotic-assisted surgery for the KA.

The field of knee prosthesis revision, exponentially growing, should also benefit from robotic surgery. The preparation of areas of bone loss will allow precise adjustment for wedges or cones, combined with careful planning of the revision prosthesis taking into account the ligament balance prior to removal of the implants to be revised.

\subsection{Case Example}

A patient of 40 years consults for painful unstable patella. She had history of recurrent patellar dislocation, which was partially improved by distalization of the anterior tibial tuberosity. The patient presented with a knee being valgus aligned but without any stiffness, a patellar maltracking and a J sign. The X-rays showed patellofemoral osteoarthritis and trochlear dysplasia type D (Dejour classification) and normal patellar height (Fig. 26.5). The tibial tuberosity-trochlear groove (TT-TG) distance, as measured on CT scan, was $35 \mathrm{~mm}$. 


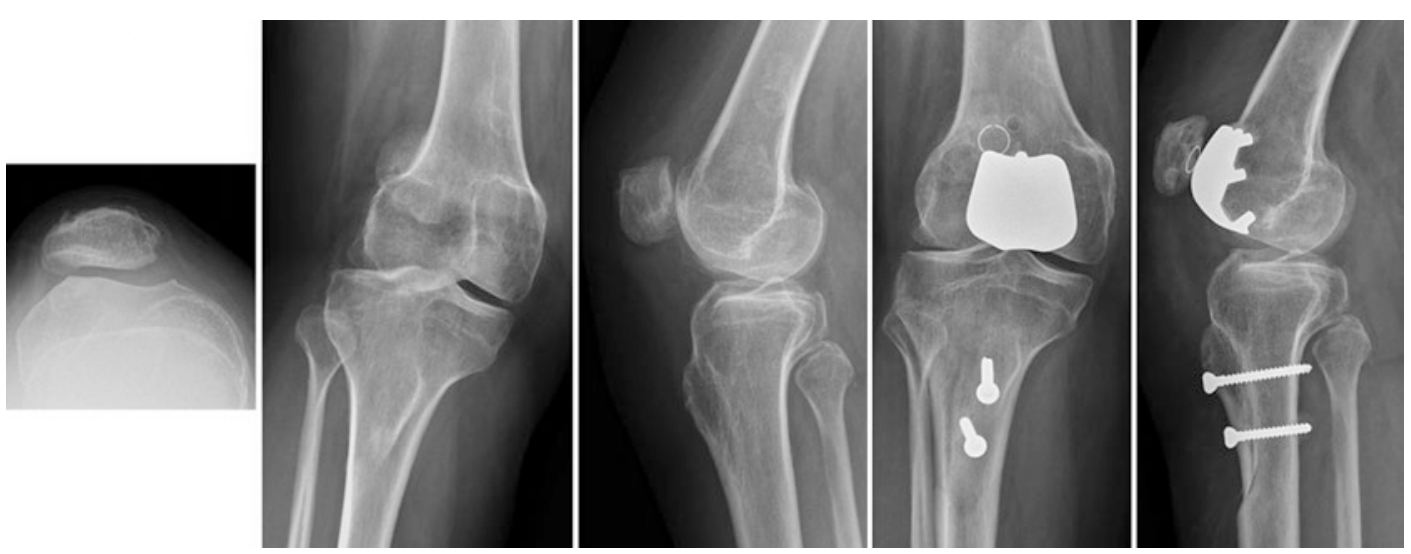

Fig. 26.5 Pre- and post-operative knee radiographs
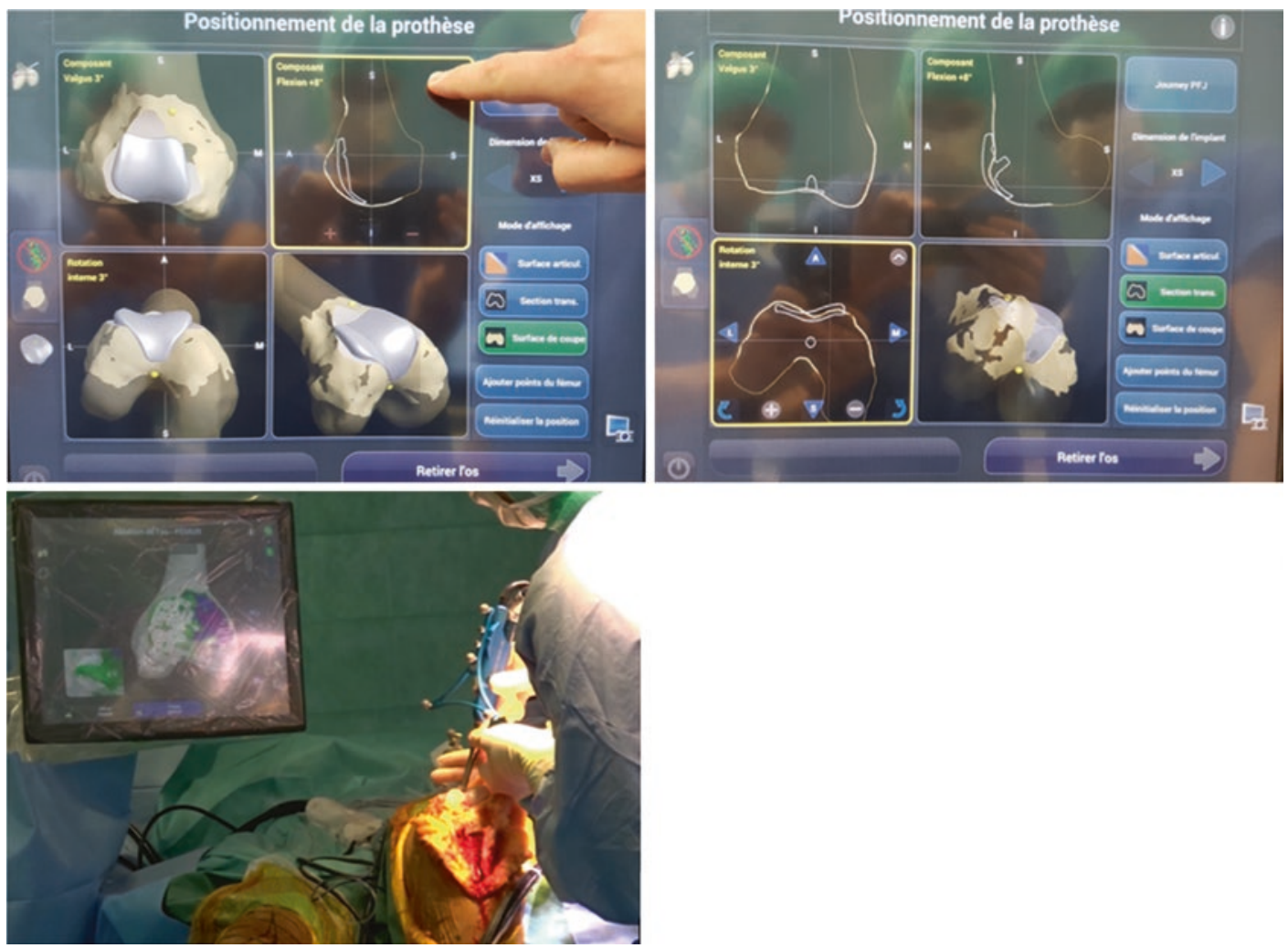

Fig. 26.6 Implant positioning with the robotic assistance before bone removal with the retro-controlled burr

She undertook a bilateral patello-femoral prosthesis associated with medialization of the anterior tibial tuberosity. The robotic assistance allowed precise implantation of the trochlea component in the three dimensions to obtain a good patellar tracking without impingement. In this indication, it is sound to lateralize a little the trochlea in order to improve the tracking and to drill sufficiently in order to avoid the increase of the constraints in the anterior compartment (Fig. 26.6). 


\section{References}

1. van der List JP, Chawla H, Joskowicz L, Pearle AD. Current state of computer navigation and robotics in unicompartmental and total knee arthroplasty: a systematic review with meta-analysis. Knee Surg Sports Traumatol Arthrosc. 2016;24:3482-95.

2. Lonner JH, Moretti VM. The evolution of imagefree robotic assistance in unicompartmental knee arthroplasty. Am J Orthop (Belle Mead NJ). 2016;45: 249-54.

3. Ponzio DY, Lonner JH. Robotic technology produces more conservative tibial resection than conventional techniques in UKA. Am J Orthop (Belle Mead NJ). 2016;45:E465-8.

4. Weber P, Schroder C, Laubender RP, et al. Joint line reconstruction in medial unicompartmental knee arthroplasty: development and validation of a measurement method. Knee Surg Sports Traumatol Arthrosc. 2013;21:2468-73.

5. Herry Y, Batailler C, Lording T, Servien E, Neyret P, Lustig S. Improved joint-line restitution in unicompartmental knee arthroplasty using a robotic-assisted surgical technique. Int Orthop. 2017;41:2265-71.

6. Bell SW, Anthony I, Jones B, MacLean A, Rowe P, Blyth M. Improved accuracy of component positioning with robotic-assisted unicompartmental knee arthroplasty: data from a prospective, randomized controlled study. J Bone Joint Surg Am. 2016;98: 627-35.

7. Marcovigi A, Zambianchi F, Sandoni D, Rivi E, Catani F. Robotic-arm assisted partial knee arthroplasty: a single centre experience. Acta Biomed. 2017;88: 54-9.

8. Pearle AD, van der List JP, Lee L, Coon TM, Borus TA, Roche MW. Survivorship and patient satisfaction of robotic-assisted medial unicompartmental knee arthroplasty at a minimum two-year follow-up. Knee. 2017;24:419-28.
9. Plate JF, Augart MA, Seyler TM, et al. Obesity has no effect on outcomes following unicompartmental knee arthroplasty. Knee Surg Sports Traumatol Arthrosc. 2017;25:645-51.

10. Gladnick BP, Nam D, Khamaisy S, Paul S, Pearle AD. Onlay tibial implants appear to provide superior clinical results in robotic unicompartmental knee arthroplasty. HSS J. 2015;11:43-9.

11. Turktas U, Piskin A, Poehling GG. Short-term outcomes of robotically assisted patello-femoral arthroplasty. Int Orthop. 2016;40:919-24.

12. Cip J, Widemschek M, Luegmair M, Sheinkop MB, Benesch T, Martin A. Conventional versus computerassisted technique for total knee arthroplasty: a minimum of 5-year follow-up of 200 patients in a prospective randomized comparative trial. J Arthroplast. 2014;29:1795-802.

13. Blakeney W, Clement J, Desmeules F, Hagemeister N, Riviere C, Vendittoli PA. Kinematic alignment in total knee arthroplasty better reproduces normal gait than mechanical alignment. Knee Surg Sports Traumatol Arthrosc. 2019;27:1410-7.

14. Courtney PM, Lee GC. Early outcomes of kinematic alignment in primary total knee arthroplasty: a metaanalysis of the literature. J Arthroplast. 2017;32:20282032.e2021.

15. Yeo JH, Seon JK, Lee DH, Song EK. No difference in outcomes and gait analysis between mechanical and kinematic knee alignment methods using robotic total knee arthroplasty. Knee Surg Sports Traumatol Arthrosc. 2019;27:2385.

16. Calliess T, Ettinger M, Savov P, Karkosch R, Windhagen $\mathrm{H}$. Individualized alignment in total knee arthroplasty using image-based robotic assistance: video article. Orthopade. 2018;47:871-9.

17. Urish KL, Conditt M, Roche M, Rubash HE. Robotic total knee arthroplasty: surgical assistant for a customized normal kinematic knee. Orthopedics. 2016;39:e822-7.

Open Access This chapter is licensed under the terms of the Creative Commons Attribution 4.0 International License (http://creativecommons.org/licenses/by/4.0/), which permits use, sharing, adaptation, distribution and reproduction in any medium or format, as long as you give appropriate credit to the original author(s) and the source, provide a link to the Creative Commons license and indicate if changes were made.

The images or other third party material in this chapter are included in the chapter's Creative Commons license, unless indicated otherwise in a credit line to the material. If material is not included in the chapter's Creative Commons license and your intended use is not permitted by statutory regulation or exceeds the permitted use, you will need to obtain permission directly from the copyright holder. 